\section{Virtually wild}

Dazzling the millions who troop past, the habitat dioramas of New York's American Museum of Natural History, including this gorilla showcase, have fused science and art intohyper-real three-dimensional displays of animals in their native savannah, swamp or plain. Windows an Nature: The Great Habitat Diomamas of the American Museum of Natural History by Stephen Christopher Quinn (Harry N. Abrams, \$40) takes a behind-the-scenes look atmore than $\mathbf{4 0}$ of them, each a testament to the explorers, naturalists, taxidermists and artists who painstakingly brought them to life more than half a century ago. This heroic task it took 18 years to complete the 29 dioramas of the North Americanmammal section alone inspired a number of conservation measures, as well as considerable awe.

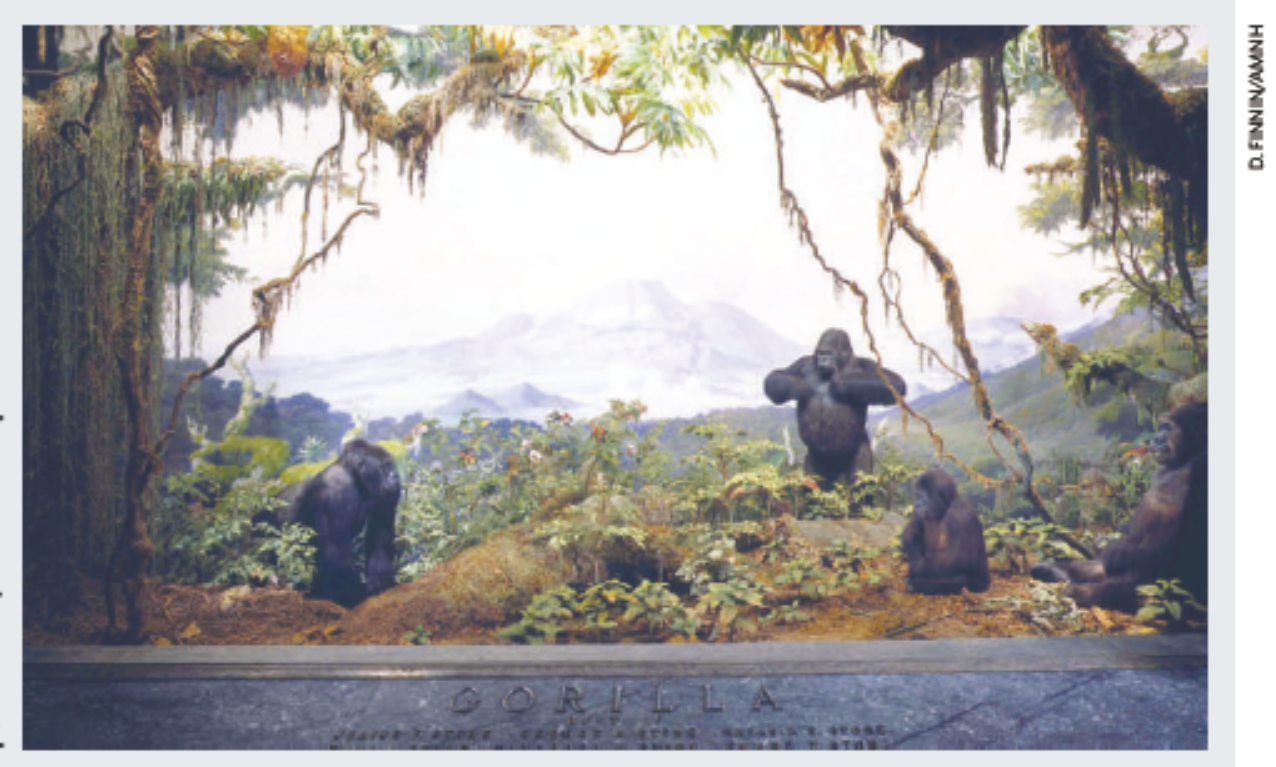

\title{
A weird, wired world
}

\author{
Entangled World: The Fascination of \\ Quantum Information and Computation \\ edited by Jürgen Audretsch \\ John Wiley: 2006. 312 pp. \\ $\$ 35, £ 22.50, € 33.80$
}

\section{Vlatko Vedral}

Quantum entanglement seems to be at the core of all the weirdness in quantum mechanics. Erwin Schrödinger called it the characteristic trait of quantum mechanics. Richard Feynman said that there was only one mystery in quantum mechanics and everything else can be seen as just its (frequently complex) consequences.

Entanglement - a bewildering set of correlations between objects in the quantum world - has historically been thought to lead to various physical, and possibly logical, paradoxes involving notions such as non-locality and cats that exist simultaneously in dead and alive states. Interestingly for engineers and computer scientists, all this quantum weirdness, far from being a hindrance, can be used to construct quantum computers that can run much faster than any current computer. Far from just being a theoretical quirk, small-scale quantum computers have been experimentally made that can perform simple calculations.

At least as interesting to physicists, although somewhat more controversial, is the issue that the very existence of the classical (nonquantum) world also crucially depends on quantum weirdness, or more specifically quantum entanglement. To put it more provocatively, the classical world behaves predictably, deterministically and in a localized way only because of the entanglement that exists on the larger scale in the Universe. This hypothesis goes under the name of decoherence, which can be thought of as synonymous with the entanglement between a physical system and its environment.

In fact, quantum computation and decoherence are two different sides of the same coin. Entanglement that can be controlled is used in quantum computers; entanglement with the environment that is uncontrollable causes decoherence, destroying quantum computers and facilitating the emergence of the classical world. The distinction between controllable or uncontrollable entanglement makes no difference to the Universe, and is only important to the tool-building human mind.

Entangled World is a nice collection of essays and reviews addressing all three of the above aspects of entanglement: the philosophical (dead-and-alive cats), the physical (decoherence) and the technological (quantum computers). This makes it a unique collection, and is its strongest selling point.

The book contains several basic yet erudite expositions, many written by people who have made significant contributions to the field, such as Reinhard Werner, Rainer Blatt, Gerhard Rempe, Harald Weinfurter and Erich Joos. The first few essays are on the basic principles of quantum mechanics, and then the book moves on to entanglement and its use in quantum computation, teleportation and other quantum wizardry. The second half of the book contains reviews on the emergence of the classical world through decoherence, concluding with an essay by Michael Esfeld on what all this really means. Physicists like me, although always wary and suspicious of philosophy and the soundness of its reasoning, still like to read such musings about the meaning of quantum physics. This is, after all, why wedo science: to understand and explain the world we live in.

My only concern about the book is that it perhaps sits somewhat uneasily between being a 'popular science' book and a third-year undergraduate introduction to the field. Some of the early essays could easily be part of a popular book, containing as they do very few equations, whereas the reviews of quantum computation and decoherence are more technical and do require some undergraduate physics training, although nothing terribly excessive. More consistency of style and level throughout the book would have been desirable, so it would totally cap tivate at least one of its target audiences.

But what about some of the really fundamental questions in quantum physics? Can large-scale quantum computers be made that would operate at room temperature? Is the world really universally quantum mechanical, or do we also need the notion of a classical world? If we do, where exactly is the division between the two worlds? Can cats (or indeed humans) really be dead and alive at the same time? Are macroscopic superpositions just a question of money and experimental ingenuity, or will they one day collapse together with quantum physics? Unfortunately, the reader is unlikely to find answers to these questions in Entangled World, or indeed in any other physics book.

What you will find in Entangled World is a comprehensible, friendly and insightful introduction into modern ideas in quantum physics, including some fascinating, albeit brief, excursions into quantum gravity. These are the salient points to further enthuse you about the subject and perhaps motivate you to do a little thinking about the big questions.

Vlatko Vedral is in the Quantum Information Group, School of Physics and Astronomy, University of Leeds, Leeds LS2 9JT, UK. 